\title{
The effects of the modified intermittent sequential pneumatic device (MISPD) on exercise performance following an exhaustive exercise bout
}

\author{
Avigdor Zelikovski MD, Clive L. Kaye* MSc, Gershon Fink*, Shimon A. Spitzer* MD and \\ Yair Shapirot MD \\ Department of Vascular Surgery and *Exercise Testing Laboratory, Beilinson Medical Center, Petah Tiqva and \\ tDepartment of Physiology and Pharmacology, Sackler School of Medicine, Tel Aviv University, Israel
}

\begin{abstract}
The Lymphapress, a pneumatic sequential intermittent device is recognized as one of the most effective conservative treatments for lymphoedema, due to its effective 'milking mechanism'. This led us to hypothesize that accelerated removal of fatigue-causing metabolites by mechanical massage could improve an athlete's performance capacity. We conducted trials with 11 men who exercised at a constant workload, on a cycle ergometer, until exhaustion. During a 20 -min recovery period a new modified pneumatic sequential intermittent device (the MISPD) was applied to the subjects' legs. The men then performed a second constant load exercise bout. Cardiorespiratory parameters were measured during exercise and blood was withdrawn during recovery for the determination of lactate, pyruvate, ammonia, bicarbonate and $\mathrm{pH}$. No difference was found in the blood levels of the 'fatigue causing metabolites' during passive recovery (PR) and recovery with the MISPD (MR). However, the MISPD effected a $45 \%$ improvement in the subjects' ability to perform the subsequent exercise bout. The accumulation of fluid in the interstitial space after exercise and its disappearance after the use of the MISPD offers one possible explanation for these results, although psychological effects cannot be discounted.
\end{abstract}

Keywords: Muscle fatigue, massage, pneumatic device, performance enhancement

Muscle fatigue has been defined as the failure to maintain the required or expected force ${ }^{1}$. For many years, physiologists have debated the origin and mechanism of muscle fatigue. Some ascribe it to the central nervous system, while others consider it to be peripheral ${ }^{2,3}$.

One of the four basic processes involved in muscle fatigue, as suggested by Simonson ${ }^{4}$, is the accumulation of metabolites. The active removal of these metabolites should therefore alleviate fatigue.

Several studies have shown that the performance capacity is greater after active than after passive recovery ${ }^{5,6}$. The removal of lactic acid from the muscles and the blood is considered to be one

Address for correspondence: C. Kaye MSc, Pulmonary Institute, Beilinson Medical Center, Petah Tiqva 49100, Israel

(C) 1993 Butterworth-Heinemann Ltd

0306-3674/93/040255-05 important aspect of the recovery process ${ }^{7-10}$. Lactic acid removal is more effective during active recovery than during passive recovery ${ }^{8,11,12}$ but there is no agreement as to the optimal intensity of the active recovery. Massage of the fatigued limbs is regarded as more effective than passive recovery, but apart from statements by athletes supporting massage, scientific research in this field is lacking ${ }^{13}$.

The Lymphapress (Mego-Afek, Kibutz Afek, Israel), a sequential pneumatic intermittent compression device, is considered to be one of the best conservative treatments for lymphoedema, due to its effective milking mechanism of the extremities ${ }^{14,15}$. We therefore hypothesized that by massaging previously fatigued legs with a new modified intermittent sequential pneumatic device (MISPD) (patent pending), especially developed for this purpose, a more rapid removal of the fatigue-causing metabolites would be achieved and this could improve the subjects' ability to perform a subsequent exercise bout.

\section{Subjects and methods}

Eleven healthy men participated in the study (of mean(s.d.) age 26.6(4.9) years, mean(s.d.) height $176.3(3.7) \mathrm{cm}$, and mean(s.d.) weight $70.7(6.5) \mathrm{kg}$ ). None were trained athletes but all regularly performed some form of physical activity. All were informed of the experimental procedure before signing a consent form. The purpose of the research was not explained to the subjects in order to minimize psychological effects.

\section{Instrumentation}

The exercise tests were performed on an electronically braked cycle ergometer (Ergoline 800, Mijnhardt bv, Bunnik, The Netherlands). During the tests, the subjects were connected to an electrocardiogram (ECG) (6353 Cardiofax Nihon Koden, Tokyo, Japan) and breathed through a tube connected to a pneumotachometer and a gas analyser module (CPX Medgraphics, Medical Graphics Corp., St Paul, Minnesota, USA). The data obtained breath by breath 
were displayed and stored with a computer (Mitsubishi MP 286, Misubishi Electronics America Inc., Torrance, California, USA).

The MISPD contains a compressor and distributor which create a continuous cycle of ascending pressure waves (of $50 \mathrm{mmHg}$ ) into sleeves which are placed on the lower limbs. The sleeves house 12 independent overlapping cells which are fed independently from the pressure source. The action of the device creates waves of pressure on the lower limbs causing a continuous milking effect.

\section{Experimental procedure}

Each subject completed two maximal incremental exercise tests, according to the protocol of Wasserman $e t$ al. ${ }^{16}$. After a 3-min period of rest, the subjects performed unloaded pedalling, maintaining a cycle speed of 60 r.p.m. The work rate was then progressively increased until the subject could no longer maintain a cycle frequency of 40 r.p.m. From the results of the best incremental test, a constant workload was calculated at $80 \%$ of the maximum $\dot{V} \mathrm{O}_{2}$ achieved in the incremental test.

The subjects were asked to refrain from any form of exercise for at least $18 \mathrm{~h}$ before each test day and were told to eat only a small breakfast, at least $2 \mathrm{~h}$ before testing. After arrival at the laboratory, each subject lay down and a catheter was securely placed in a superficial forearm vein. Blood samples were taken and analysed for lactate, pyruvate, $\mathrm{pH}$, bicarbonate and ammonia. The subjects then mounted the cycle ergometer, were connected to the testing equipment and rested for $3 \mathrm{~min}$ before performing exercise bout 1 (constant workload) until exhaustion. After a 20-min rest the subjects exercised again at the same constant workload until exhaustion. One week later the subjects performed the same test again. They were divided into two groups - group 1 (six subjects) rested in a supine position after bout 1 . One week later the MISPD was applied during the rest period. Group 2 (five subjects) performed the same test but in a different order, i.e. the MISPD was applied after the first exercise bout but not during the rest after bout 1 a week later. The subjects did not know before the test day which group they were in.

Blood samples were drawn at rest before bout 1 and every 2 min during recovery after bout 1 . All the relevant cardiorespiratory parameters were measured before, and during, the exercise bouts.

\section{Chemical analysis}

Blood lactate and pyruvate were determined enzymatically using protein-free filtrates. The reaction was monitored by following the consumption (for pyruvate) or the formation (for lactate) of NADH at $340 \mathrm{~nm}^{17}$. The concentration of ammonia was determined using the Sigma diagnostic method ${ }^{18}$ (Sigma Chemical Co., St Louis, Missouri, USA). The $\mathrm{pH}$ and bicarbonate were measured using the $\mathrm{pH} /$ blood gas analyser system IL $6002^{19}$ (Instrumentation Laboratories International, Lexington, Massachusetts, USA).

\section{Statistical analysis}

The data were evaluated using two-way analysis of variance with repeated measures, and paired $t$ tests where applicable. An $\alpha$ level of 0.05 was chosen.

\section{Results}

The mean(s.d.) maximum oxygen consumption $\left(\dot{V}_{\mathrm{O}_{2}}\right)$ achieved by subjects in the maximal incremental aerobic capacity tests was $48(4) \mathrm{ml} \mathrm{kg}^{-1} \mathrm{~min}^{-1}$. The mean(s.d.) constant workload for the exercise bout was 211(16) W. No significant difference was found between group 1 and group 2 for any of the parameters tested.

The mean resting values of the cardiorespiratory parameters are summarized in Table 1 . The values at rest before bout 2 were higher than those before bout 1 but the use of the MISPD had no significant effect on these values and they remained unchanged.

The mean $\dot{V}_{\mathrm{O}_{2}}$ and carbon dioxide production $\left(\dot{V} \mathrm{CO}_{2}\right)$ achieved in exercise bout 2 were slightly lower when compared with these values in bout 1 but there was no significant difference between the two treatment groups (Table 2). The subjects managed to achieve a higher mean maximum heart rate (HR) in bout 2 after treatment with the MISPD compared with that achieved in bout 2 after passive recovery $(P$ $<0.001)$. The subjects also achieved a higher mean maximum heart rate in bout 2 than in bout 1 after treatment with the MISPD $(P<0.05)$. After passive recovery the mean maximum heart rate was slightly reduced, although not significantly. The subjects managed to achieve a higher mean maximum

Table 1. Mean(s.d.) values of cardiopulmonary parameters at rest before exercise bouts 1 and 2

\begin{tabular}{|c|c|c|c|c|}
\hline \multirow[b]{2}{*}{ Parameter } & \multicolumn{2}{|c|}{ Bout 1} & \multicolumn{2}{|c|}{ Bout 2} \\
\hline & $(P R)$ & $(M R)$ & $(P R)$ & $(M R)$ \\
\hline $\begin{array}{l}\text { HR }\left(\text { beats } \min ^{-1}\right) \\
\dot{\mathrm{VE}}\left(\mathrm{Imin}^{-1}\right) \\
\dot{\mathrm{V}} \mathrm{O}_{2}\left(\mathrm{ml} \mathrm{min}^{-1}\right) \\
\dot{V} \mathrm{CO}_{2}\left(\mathrm{ml} \mathrm{min}^{-1}\right)\end{array}$ & $\begin{array}{l}72(11) \\
15(3) \\
375(77) \\
358(62)\end{array}$ & $\begin{array}{c}71(8) \\
15(4) \\
337(50) \\
345(53)\end{array}$ & $\begin{array}{l}104(12) \\
21(7) \\
554(111) \\
416(121)\end{array}$ & $\begin{array}{c}106(15) \\
20(3) \\
550(110) \\
414(104)\end{array}$ \\
\hline
\end{tabular}

$P R$, recovery period passive; $M R$, recovery period with the modified intermittent sequential pneumatic device (MISPD); HR, mean maximum heart rate; $\dot{V E}$, maximum ventilation. The mean values at rest before bout 2 were significantly higher than those before bout 1 , but the MISPD had no effect on these values. $P$ was always $<0.05$ using paired $t$ test

Table 2. Mean(s.d.) maximum values of cardiopulmonary parameters achieved during exercise bouts 1 and 2

\begin{tabular}{|c|c|c|c|c|}
\hline \multirow[b]{2}{*}{ Parameter } & \multicolumn{2}{|c|}{ Bout 1} & \multicolumn{2}{|c|}{ Bout 2} \\
\hline & $(P R)$ & $(M R)$ & $(P R)$ & $(M R)$ \\
\hline $\begin{array}{l}H R\left(\text { beats } \min ^{-1}\right) \\
\dot{V} \mathrm{E}\left(\mathrm{I} \min ^{-1}\right) \\
\dot{V} \mathrm{O}_{2}\left(\mathrm{ml} \mathrm{min} \min ^{-1}\right) \\
\dot{V} \mathrm{CO}_{2}\left(\mathrm{ml} \min ^{-1}\right)\end{array}$ & $\begin{array}{c}197(9) \\
134(17) \\
3461(319) \\
3640(249)\end{array}$ & $\begin{array}{c}194(14) \\
130(23) \\
3427(279) \\
3668(306)\end{array}$ & $\begin{array}{c}194(10) \\
125(21) \\
3259(318) \\
3355(271)\end{array}$ & $\begin{array}{c}202(8) \\
135(16) \\
3319(275) \\
3436(330)\end{array}$ \\
\hline
\end{tabular}

$H R$, mean maximum heart rate; $\dot{V} \mathrm{E}$, maximum ventilation 
ventilation ( $\dot{\mathrm{VE}})$ in bout 2 after treatment with the MISPD compared with that achieved in bout 2 after passive recovery $(P<0.05)$. After passive recovery, the mean maximum ventilation was significantly reduced $(P<0.005)$.

The mean(s.d.) basal metabolite values before exercise were all within the normal range (lactate $1.3(0.2) \mathrm{mmoll}^{-1}$, pyruvate $48(12) \mu \mathrm{moll}^{-1}$, ammonia 24(4) $\mathrm{moll}^{-1}$, bicarbonate 23(2) $\mathrm{mmoll}^{-1}, \quad \mathrm{pH}$ 7.41(0.3))

The blood metabolite data are summarized in Figure 1. Blood lactate, $\mathrm{pH}$, bicarbonate and ammonia were not significantly different during passive and MISPD recovery. The mean pyruvate concentration during passive recovery was higher than during recovery with the MISPD $(P<0.05)$ (Figure 2$)$.
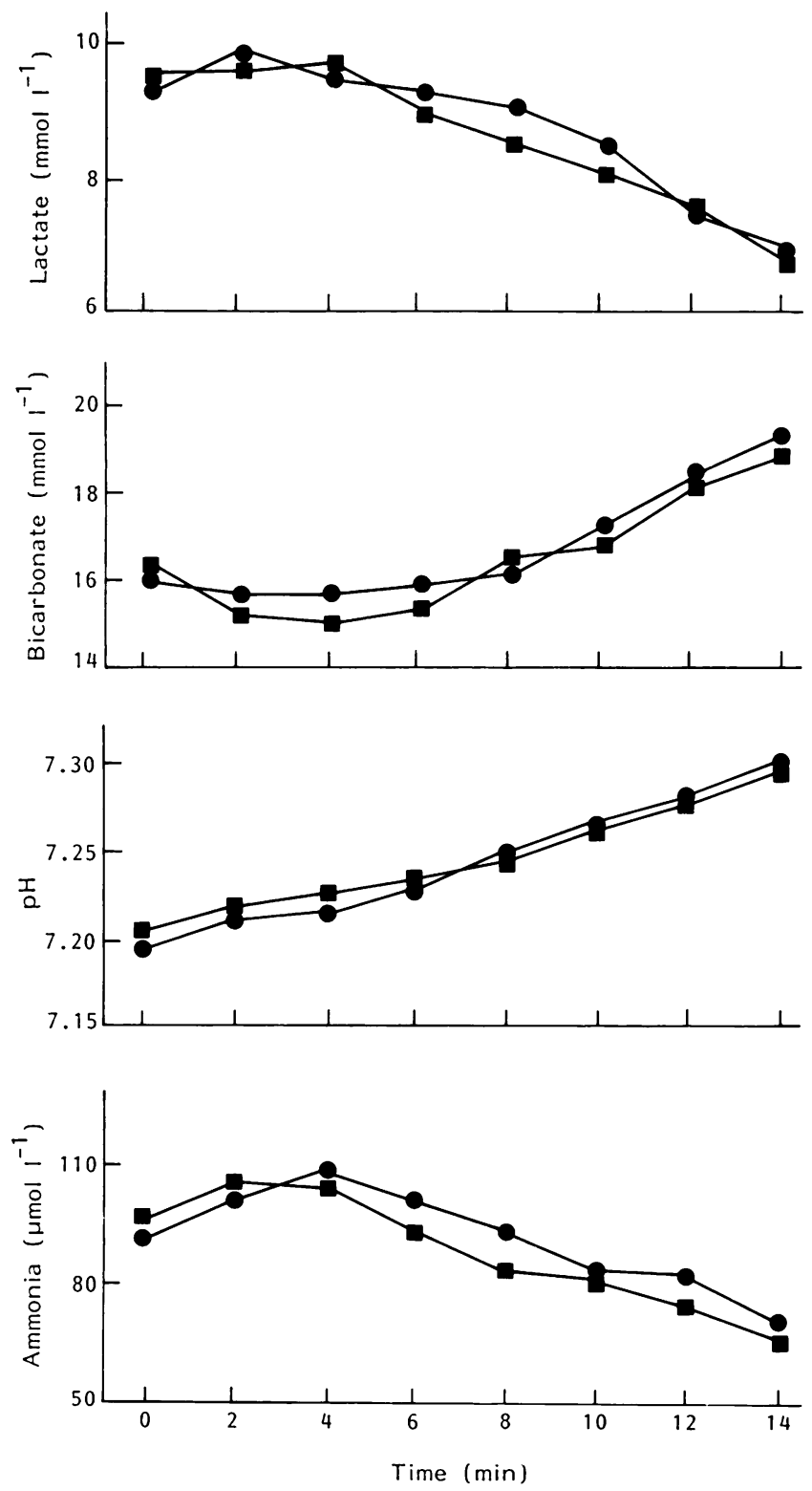

Figure 1. Mean values of blood lactate, $\mathrm{pH}$, bicarbonate and ammonia during passive recovery $(\square, P R)$ and recovery with the MISPD (O, MR). No significant difference was found in these blood metabolite levels during $P R$ and $M R$
Table 3. Mean(s.d.) performance time of bouts 1 and 2

\begin{tabular}{lllll}
\hline Condition & $\begin{array}{c}\text { Bout 1 } \\
(\mathrm{min})\end{array}$ & $\begin{array}{c}\text { Bout 2 } \\
(\mathrm{min})\end{array}$ & Difference & $P$ \\
\hline Passive rest (PR) & $11.6(1)$ & $6.4(0.7)$ & 5.3 & $<0.0005$ \\
MISPD (MR) & $10.9(0.8)$ & $8.7(0.8)$ & 2.2 & $<0.0001$ \\
\hline
\end{tabular}

$P R$, recovery period passive; $M R$, recovery period with the modified intermittent sequential pneumatic device (MISPD). The performance time of bout 2 after treatment with the MISPD was significantly higher than that after passive recovery, $P<0.004 P R$ versus MR; paired $t$ test used

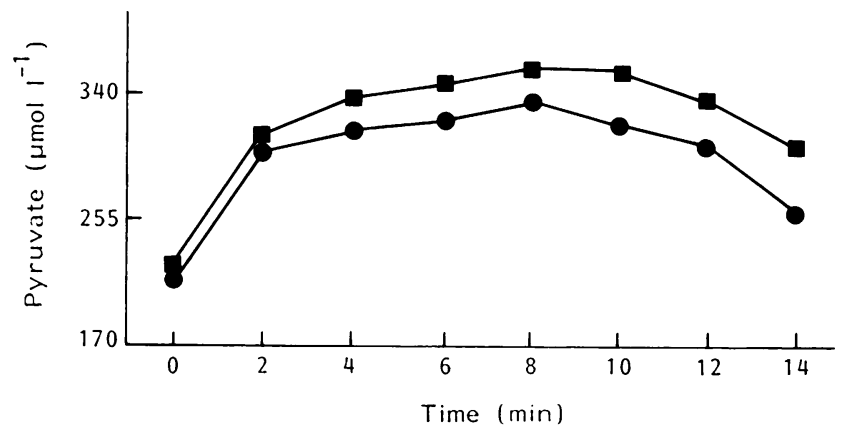

Figure 2. Mean values of blood pyruvate concentrations during passive recovery $(\square, P R)$ and recovery with the $\operatorname{MISPD}(\mathbf{O}, \mathrm{MR}) P<0.05$

The mean performance times for bouts 1 and 2 are summarized in Table 3. No significant difference was found in the duration of bout 1 , between the two groups. There was a reduction in the performance time of bout 2, but after recovery with the MISPD the subjects performed for $80 \%$ of the duration of bout 1 compared with $55 \%$ in bout 2 after passive recovery. The performance time of bout 2 after treatment with the MISPD was significantly higher than that after passive recovery $(P<0.004)$. It is important to point out that this finding was consistent and occurred in all the subjects tested.

\section{Discussion}

Muscle fatigue is a complex phenomenon involving biochemical, physiological and psychological elements ${ }^{2}$. Several basic mechanisms have been proposed for the fatigue process ${ }^{4}$. One of these is the accumulation of metabolites causing fatigue in the working muscles ${ }^{2,20}$.

During the anaerobic phase of exercise, lactic acid and other metabolites are produced in the muscle and are released into the blood. Theoretically, any means that enhance the removal of the fatigue-causing metabolites (lactic acid and ammonia), from the working muscles should improve a subsequent exercise performance. In this study the MISPD was used to improve the subjects' performance in a subsequent exercise bout. The original rationale behind this experiment was that the continuous milking effect of the MISPD on the leg muscles after strenuous effort, would accelerate the removal of metabolites from the legs and thus improve the 
performance of the subjects in a subsequent exercise bout. The results demonstrated a significant improvement in the subjects' performance in the subsequent bout after recovery with the use of the MISPD, manifested by a prolongation of exercise duration by $45 \%$.

The metabolic blood data, however, failed to show a corresponding change in the blood levels of the metabolites measured. The MISPD apparently does not achieve an accelerated removal of metabolites from the muscles as determined from their levels in peripheral venous blood. The possibility that peripheral venous blood does not always reflect muscle metabolic changes has been proposed by several investigators ${ }^{21-23}$. Peripheral venous blood, however, has been used to reflect the effectiveness of different recovery protocols ${ }^{10,24,25}$. Accordingly, the accelerated removal of metabolites with the MISPD from the exercised muscles should be reflected in venous blood concentrations. An exceptional observation was the lower pyruvate levels during recovery with the MISPD after bout 1, compared with passive recovery. The mechanism of this observation is not clear. It is possible that there is a reduced production of pyruvate in the muscle due to its slower conversion from lactate or there is a higher metabolism of pyruvate in the muscle. Another possibility is that the pyruvate is more effectively removed from the blood during recovery with the MISPD.

The fact that the subjects significantly improved their performance time in bout 2 after treatment with the MISPD and also managed to achieve a higher mean maximum ventilation and a higher mean maximum heart rate, indicates that they were better able to overcome the peripheral fatigue of their legs.

When considering other (non-metabolic) causes that could explain our results, one should note that the Lymphapress is regarded as one of the best conservative treatments for lymphoedema of the limbs, due to its effective milking mechanism ${ }^{14,26}$. Since the Lymphapress also reduces the water content of the limb ${ }^{26}$, it is possible to propose a different mechanism for the action of the MISPD. According to this hypothesis the MISPD, which was constructed for use on normal lower limbs, may prevent the formation of fluid in the interstitial space, a factor which could explain our results. Several studies have shown that during and after exercise there is an accumulation of fluid in the muscle ${ }^{27,28}$. Sejersted ${ }^{27}$ and Sjogaard ${ }^{28}$ found that at the end of exercise, fluid accumulates in the muscles and the interstices. This is due to an increase in blood flow to the working muscle along with an increase in blood pressure, as well as an increase in the osmotic pressure of the tissues from the accumulation of metabolites. During exercise the contraction of the muscles helps to reduce the increase of fluid in the interstices.

The accumulation of fluid in the interstices can cause several effects: an increase in the diffusion distance between the capillaries and the muscle cells, changes in membrane characteristics and an increase in pressure due to an increase in fluid $^{27-29}$.

Each of these can cause a disturbance in the membrane function and a consequent disturbance in muscle function. The effective removal of the excess fluid that accumulates in the interstitial space after exercise could, therefore, prevent the disturbance in muscle function before bout 2 thus causing the improved performance of the subjects in bout 2 . Our present study does not present data to support this hypothesis. This could be verified by the determination of plasma volume changes after using the MISPD. Psychological reasons for improved performance cannot be ruled out. The massaging action of the MISPD creates a pleasant sensation on the muscles to which it is applied. It is therefore possible that the subjects felt better prepared for the subsequent exercise bout and because of this they managed to exercise for a longer duration following application of the MISPD. Diverting activities carried out during the rest period following an exercise bout have been shown to be effective in improving subjects' ability to perform a subsequent exercise bout ${ }^{6}$. This was attributed to an effect of the central nervous system.

Although we could not elucidate the mechanism of its action, treatment with the MISPD caused a $45 \%$ improvement in the subjects' ability to perform a subsequent exercise bout.

Further investigation is required in order to acquire a better understanding of the mode of action of the MISPD and its effect on muscle fatigue.

\section{Acknowledgement}

Submitted by C. Kaye in partial fulfillment of the requirements for the Master's Degree in Physiology, Tel Aviv University, Israel.

\section{References}

1 Edwards RHT. Human muscle function and fatigue. In: Human Muscle Fatigue. CIBA Foundation Symposium, 82. London, UK: Pitman, 1981: 1-18.

2 Astrand PO, Rodahl K. Textbook of Work Physiology. New York, USA: McGraw-Hill, 1986.

3 Vollestod NK, Sejersted OM. Biochemical correlates of fatigue. Eur J Appl Physiol 1988; 57: 336-47.

4 Simonson E. Physiology of Work Capacity and Fatigue. Springfield, Illinois, USA: Thomas, 1971.

5 Alpert JS. The mechanism of increased maximum work performance of small muscle groups resulting from 'diverting work' with other muscle groups. Acta Physiol Scand 1969; 77: 261-71.

6 Asmussen E, Mazin B. Recuperation after muscular fatigue by 'diverting activities'. Eur J Appl Physiol 1978; 38: 1-7.

7 Belcastro $\mathrm{AN}$, Bonen A. Lactic acid removal rates during controlled and uncontrolled recovery exercise. J Appl Physiol 1975; 39: 932-6.

8 Boileau RA, Misner JE, Dykstra GL, Spitzer TA. Blood lactic acid removal during treadmill and bicycle exercise at various intensities. I Sports Med 1983; 23: 159-67.

9 Rontoyannis GP. Lactate elimination from the blood during active recovery. J Sports Med 1988; 28: 115-23.

10 Weltman A, Stamford BA, Fulco C. Recovery from maximal effort exercise. Lactate disappearance and subsequent performance. J Appl Physiol Respirat Environ Exerc Physiol 1979; 47: 677-82.

11 Bulbulian R, Darabos B, Nauta S. Supine rest and lactic acid removal following maximal exercise. J Sports Med 1987; 27: 151-6.

12 McArdle WD, Katch FI, Katch VL. Exercise Physiology, Energy, Nutrition and Human Performance. 2nd ed. Pennsylvania, Philadelphia, USA: Lea and Febiger, 1986.

13 Dittmer D. Sports massage. Sports Med Info 1987; 6(2): 1-6.

14 Zelikovski A, Deutsch A, Reiss R. The sequential pneumatic compression device in surgery for lymphedema in the limbs. J Cardiovasc Surg 1983; 24: 122-6. 
15 Zelikovski A, Manoach M, Giler S, Urca I. Lympha press, a new pneumatic device for the treatment of lymphedema of the limbs. Lymphology 1980; 13: 68-73.

16 Wasserman K, Hansen JE, Sue DY, Whipp BJ. Principles of Exercise Testing and Interpretation. Pennsylvania, Philadelphia, USA: Lea and Febiger, 1987.

17 Marbach EP, Weil MH. Rapid enzymatic measurement of blood lactate and pyruvate. Clin Chem 1967; 13: 314-25.

18 Sigma Diagnostics. Ammonia Quantitative Enzymatic Determination in Plasma at $340 \mathrm{~nm}$ (Procedure no. 170-UV). St Louis, USA, Sigma Chemical Company 1986.

19 Instrumental Laboratory Operator's Manual IL System 1302 $\mathrm{pH} /$ Blood gas Analyzer: Lexington, Massachusetts, USA.

20 Hermansen L, Osnes JB. Blood and muscle $\mathrm{pH}$ after maximal exercise in man. I Appl Physiol 1972; 32: 304-8.

21 Karlsson J. Pyruvate and lactate ratios in muscle tissue and blood during exercise in man. Acta Physiol Scand 1971; 81: 455-8.

22 Knuttgen HG, Saltin B. Muscle metabolites and oxygen uptake in short-term submaximal exercise in man. J Appl Physiol 1972; 32: 690-4.
23 Shephard RJ. Physiology and Biochemistry of Exercise. New York, USA: Praeger, 1982.

24 Stamford BA, Weltman A, Moffatt R, Sady S. Exercise recovery above and below anaerobic threshold following maximal work. J Appl Physiol: Respir Environ Exerc Physiol 1981; 51: 840-4.

25 Weltman A, Regan JD. Prior exhaustive exercise and subsequent maximal constant load exercise performance. Int $J$ Sports Med 1983; 4: 184-9.

26 Wolfe JHN. Treatment of lymphedema. In: Rutherford RB, ed. Vascular Surgery. 2nd ed. Pennsylvania, Philadelphia, USA: WB Saunders, 1984: 1463-5.

27 Sejersted OM, Vollestad NK, Medbo JI. Muscle fluid and electrolyte balance during and following exercise. Acta Physiol Scand 1986; 128 (Suppl 556): 119-27.

28 Sjogaard G, Saltin B. Extra- and intracellular water spaces in muscles of man at rest and with dynamic exercise. $A m J$ Physiol (Regulatory Integrative Comparative Physiology) 1982; 243: R271-R280.

29 Sjogaard G, Savard G, Juel C. Muscle blood flow activity and its relation to muscle fatigue. Eur J Appl Physiol 1988; 57: 327-35.

\section{BIOMECHANICS IN SPORTS MEDICINE}

\section{UNIVERSITY OF SALFORD}

\section{9-30 MARCH 1994}

The rehabilitation special interest group of the BES and IPSM has combined with BASM (North West) to organise a multidisciplinary conference on the role of biomechanics in the various aspects of sports medicine.

It will run as a series of sessions on selected topics within sports biomechanics led and coordinated by acknowledged experts drawn from sports medicine, bioengineering, sports biomechanics and the paramedical professions. Two of these sessions will be available for oral presentation by delegates on any topic of relevance to sports biomechanics. The session titles are:

- Biomechanics in the prevention and diagnosis of sports injuries

- Biomechanics in the treatment of sports injuries

- Biomechanics of the sports shoe

- Training of the sports medicine professional

For further information, please contact: Carole Atkinson, The Brian Blatchford Building, University College Salford, Salford M6 6PU. Tel: 0617453365 . Fax: 0617361579. 